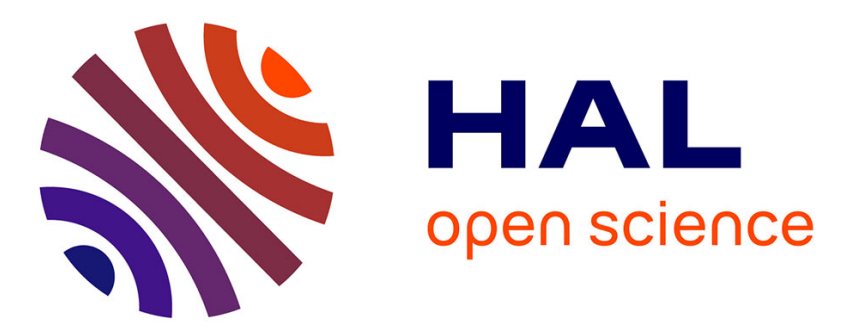

\title{
Asymmetric magnetization reversal in dipolarly coupled spin valve structures with perpendicular magnetic anisotropy
}

M Gottwald, M. Hehn, D Lacour, Thomas Hauet, F Montaigne, S Mangin, P Fischer, M.-y Im, Arjan Berger

\section{To cite this version:}

M Gottwald, M. Hehn, D Lacour, Thomas Hauet, F Montaigne, et al.. Asymmetric magnetization reversal in dipolarly coupled spin valve structures with perpendicular magnetic anisotropy. Physical Review B: Condensed Matter and Materials Physics (1998-2015), 2012, 10.1103/PhysRevB.85.064403 . hal-01345391

\section{HAL Id: hal-01345391 \\ https://hal.science/hal-01345391}

Submitted on 13 Jul 2016

HAL is a multi-disciplinary open access archive for the deposit and dissemination of scientific research documents, whether they are published or not. The documents may come from teaching and research institutions in France or abroad, or from public or private research centers.
L'archive ouverte pluridisciplinaire HAL, est destinée au dépôt et à la diffusion de documents scientifiques de niveau recherche, publiés ou non, émanant des établissements d'enseignement et de recherche français ou étrangers, des laboratoires publics ou privés. 


\title{
Asymmetric magnetization reversal in dipolarly coupled spin valve structures with perpendicular magnetic anisotropy
}

\author{
M. Gottwald, ${ }^{*}$ M. Hehn, D. Lacour, T. Hauet, F. Montaigne, and S. Mangin \\ Institut Jean Lamour, UMR CNRS 7198, Nancy-Université, BP 239, F-54506 Vandoeuvre lès Nancy, France \\ P. Fischer and M.-Y. Im \\ Center for X-ray Optics, Lawrence Berkeley National Laboratory, Berkeley, California 94720, USA \\ A. Berger \\ CIC nanoGUNE Consolider, E-20018 Donostia-San Sebastián, Spain \\ (Received 23 November 2011; revised manuscript received 13 January 2012; published 3 February 2012)
}

\begin{abstract}
Magnetization reversal has been studied in a $\mathrm{Co}_{74} \mathrm{~Tb}_{26} / \mathrm{Cu} / \mathrm{Co}_{88} \mathrm{~Tb}_{12}$ system, which is a perpendicularly magnetized ferrimagnetic bilayer separated by a nonmagnetic layer. The $\mathrm{Co}_{88} \mathrm{~Tb}_{12}$ (soft) layer (SL) exhibits a switching field much lower than the $\mathrm{Co}_{74} \mathrm{~Tb}_{26}$ (hard) layer (HL), which enabled us to study the magnetization reversal of the SL for different magnetic states of the HL. We found an asymmetric hysteresis loop for the $\mathrm{SL}$, when the HL is not fully saturated. Using careful analysis of magnetic force microscopy measurements we conclude that the asymmetry originates from magnetic domains formed in the HL that affect the SL reversal. A simple model based on magnetic dipolar interaction between the two layers is found to be in good agreement with the experimental data.
\end{abstract}

DOI: 10.1103/PhysRevB.85.064403

PACS number(s): $75.70 . \mathrm{Kw}, 75.70 . \mathrm{Cn}$

\section{INTRODUCTION}

The magnetization reversal process in ferromagnetic (FM) materials generally follows time-reversal symmetry with respect to the magnetic field sign. However, there are various examples for which the magnetization reversal process during ascendant field branch differs from the descendent field branch. The most famous example is the antiferromagnetic (AF)/FM system, which exhibit an "exchange-bias" effect that leads to a field axis shift of the FM hysteresis loop. ${ }^{1,2}$ Moreover additional asymmetries in the FM hysteresis loop have been observed indicating different FM reversal processes for both branches. ${ }^{3,4}$ However other systems exhibit this type of asymmetry as well. ${ }^{5-7}$ Common to all cases is that the origin of the asymmetry can be traced back to coupling phenomena, i.e., either an exchange coupling or a dipolar coupling. ${ }^{6-12}$

Dipolar couplings have attracted strong interest both for fundamental reasons and potential applications. Examples for the former are dipolar stray field coupling between nanomagnets sometimes leading to fascinating properties like geometrical frustration, ${ }^{7}$ the occurrence of multidomain formation, ${ }^{9}$ and domain wall (DW) pinning, ${ }^{10}$ which allow investigating the competition between short-range and longrange interaction models. On the other hand, dipolar fields play a major role in bit-patterned media recording, ${ }^{13}$ multilevel magnetic recording, ${ }^{14}$ and the reliability of magnetoelectric devices. ${ }^{15,16}$ For magnetization control either by field or polarized current, multilayers composed of two perpendicularly magnetized FM separated by a nonmagnetic decoupling layer are among the most studied systems..$^{9,10,13,16-21}$ When soft (SL) and hard layers (HL) have similar intrinsic domain sizes, the stray fields induced by a non-uniformly magnetized HL lead to mirrored domains in the SL during field cycles. ${ }^{18}$ However, an even more complex behavior can occur when the SL and the HL are designed differently. ${ }^{12}$ For example, in Refs. 9 and 17, asymmetric minor loops have been reported in perpendicular magnetized multilayers.

In this paper we study the physical origin of minor loop asymmetries in spin valve structures with perpendicular magnetization. Characterization is done using magnetometry measurements and magnetic force microscopy (MFM) analysis of the microscopic magnetic structure within the films. A general model of asymmetric dipolar coupling for perpendicularly magnetized multilayer is developed, which explains well our experimental data.

\section{SAMPLES}

$\mathrm{Si} / \mathrm{Ta}(5 \mathrm{~nm}) / \mathrm{Co}_{74} \mathrm{~Tb}_{26}(20 \mathrm{~nm}) / \mathrm{Cu}(3.5 \mathrm{~nm}) / \mathrm{Co}_{88} \mathrm{~Tb}_{12}(20$ $\mathrm{nm}) / \mathrm{Cu}(2 \mathrm{~nm}) / \mathrm{Pt}(2 \mathrm{~nm})$ multilayers were grown by $\mathrm{DC}$ magnetron sputtering with base pressure less than $5 \times$ $10^{-9}$ mbar. The $\mathrm{Co}_{1-\mathrm{x}} \mathrm{Tb}_{\mathrm{x}}$ alloy has been chosen due to its high perpendicular magnetic anisotropy over large values of $x$ and the possibility to easily tune the coercive field by changing $x$. Pure $\mathrm{Co}$ and $\mathrm{Tb}$ targets were used for a co-sputtering process, and the relative atomic concentration of the two elements $\mathrm{Co}$ and $\mathrm{Tb}$ was controlled by the relative sputtering power. Samples were deposited on Si (100) substrates covered first with a 5-nm-thick Ta seed layer. The multilayer is protected by a Cu/Pt bilayer. The choice of $x=12 \%$ for the SL and $x=26 \%$ for the HL ensures a large difference in the coercive fields of the layers with a well-defined antiparallel magnetization plateau in the field loop [Fig. 1(a)]. When the HL is fully saturated, the SL minor loop is symmetric with respect to zero field, indicating the absence of interlayer coupling such as exchange coupling or RKKY-like and orange-peel coupling through the $\mathrm{Cu}$ interlayer [Fig. 1(b)]. However, when the HL is not fully saturated, i.e., magnetic domains are present, asymmetric SL minor loops are observed [Fig. 1(c)]. We will show in the following that localized non-uniform stray fields are the cause of 

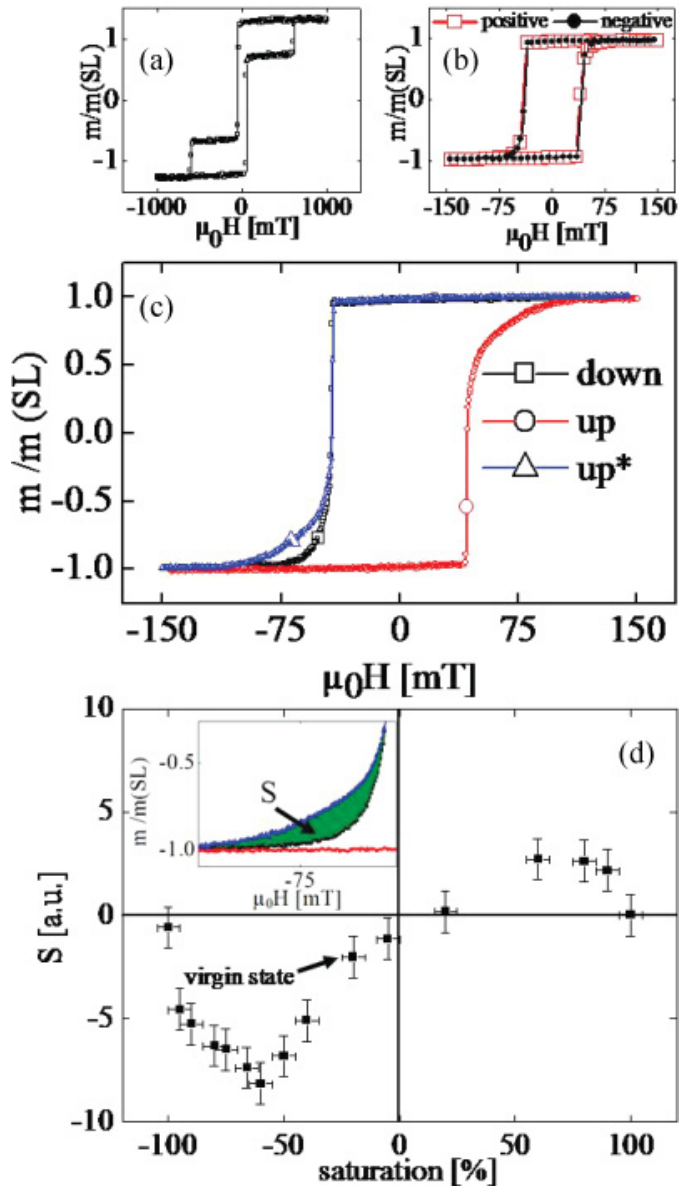

FIG. 1. (Color online) (a) Full loop of a $\mathrm{Co}_{74} \mathrm{~Tb}_{26}(20 \mathrm{~nm}) /$ $\mathrm{Cu}(3.5 \mathrm{~nm}) / \mathrm{Co}_{88} \mathrm{~Tb}_{12}(20 \mathrm{~nm})$ spin valve. (b) Minor loops for positive and negative saturated HL. (c) Typical minor loop of the SL when HL is not saturated. The blue curve is the positive branch of the loop (red curve) inverted with respect to zero field, superposed on the negative branch of the loop (black curve) to show the loop asymmetry. (d) Integrated asymmetry $S$ between down and up reversal (see inset: green area between blue and black curve) as a function of HL saturation.

the difference between the two branches. These stray fields are generated by DWs and penetrate into the neighboring layers.

\section{MAGNETOMETRY}

Since the $\mathrm{Co}_{74} \mathrm{~Tb}_{26} \mathrm{HL}$ is difficult to demagnetize through field cycling, the coupling between SL and HL has been studied by starting from HL virgin state and then moving toward positive or negative field saturation. This virgin state could not be re-established once the sample was saturated. The sample was therefore cut into several pieces that allowed us to start our experiments from equivalent initial magnetic states. The HL domain structure has been shown to remain unaffected by SL field cycling $\pm 200 \mathrm{mT}$.

The asymmetry in between ascending and descending magnetization reversal has been studied as a function of HL magnetization starting from the HL virgin state. Hereby we define the quantity of saturation as the $m_{\mathrm{HL}} / m_{\mathrm{HL}}$ (sat)ratio where $m_{\mathrm{HL}}$ is the out-of-plane component of the HL magnetization and $m_{\mathrm{HL}}$ (sat) is the saturated HL magnetization. In the so-called virgin state, the sample has a saturation ratio of about $-20 \pm 5 \%$. This nonzero value can be explained by the existence of the magnetron stray field acting on the sample during the sputtering process. We define the negative field direction along the normal to the film as parallel to the magnetization of the virgin state. Furthermore we define a reversal of the SL magnetization from antiparallel to the virgin magnetization of the HL as an "up" reversal. The inverse case is called a "down" reversal.

In Fig. 1(c) a superposition of the up and the down reversal process for a HL saturation of $-80 \%$ is shown. The field that is required to saturate the SL during the up reversal is larger than the field required during a down reversal, reflecting some kind of AF coupling between the layers. In contrast the nucleation field seems to be nearly identical for up and down reversal. One can conclude from this that the dipolar field created by the domains in the HL hardly affects the nucleation process, whereas this field influences the propagation of DWs in the SL. It seems that the dipolar stray field from the HL creates pinning potentials in the SL which are not symmetrical with regards to the sign of the external magnetic field. These pinning potentials due to the HL domains have to be at least of similar strength as the structural pinning potentials in the SL, otherwise the hysteresis loops would not be affected by them. We quantified the asymmetry by the area $S$ of the $M(H)$ profile between the up and down reversal branches [inset Fig. 1(d)] and called it integrated asymmetry. This value is equal to zero when the SL minor loop is symmetric. We calculate $S$ by

$$
S=\int_{-150 \mathrm{mT}}^{-30 \mathrm{mT}}\left[\mathrm{up}^{*}-\operatorname{down}\right] d\left(\mu_{0} H\right) .
$$

The integrated asymmetry value $S$ versus $m_{\mathrm{HL}} / m_{\mathrm{HL}}$ (sat) is plotted in Fig. 1(d). It is close to zero when $m_{\mathrm{HL}} / m_{\mathrm{HL}}$ (sat) equals 0 and $\pm 100 \%$. The integrated asymmetry reaches its largest level for $m_{\mathrm{HL}} / m_{\mathrm{HL}}$ (sat) value around $\pm 60 \%$. This observation suggests a correlation between the SL minor loop asymmetry and the domain structures in the HL. Note that starting from the virgin state the integrated asymmetry $S$ is behaving differently while saturating the $\mathrm{HL}$ in one or the other direction. This is due to the asymmetry of the virgin state, which is explained previously as due to the existence of the magnetron stray field acting on the sample during the sputtering process.

\section{MFM AND MODELING}

For the purpose of developing an understanding of these SL minor loop asymmetries, spatial distribution of HL-induced stray fields acting on the SL have been mapped out using MFM measurements (Fig. 2). At remanence, the HL exhibits a nonuniform domain structure that evolves differently for a positive and negative applied field [Figs. 2(b) and 2(c)]. This difference in HL magnetic domain structure explains the difference of amplitude for the integrated asymmetry maximum with positive or negative $m_{\mathrm{HL}} / m_{\mathrm{HL}}$ (sat), reported in Fig. 1(d). More importantly, the domain size of the smallest HL domains does not change significantly with the applied field, and its size is between 0.1 and $2 \mu \mathrm{m}$. Applying a field only modifies their spatial distribution and especially their local density. 


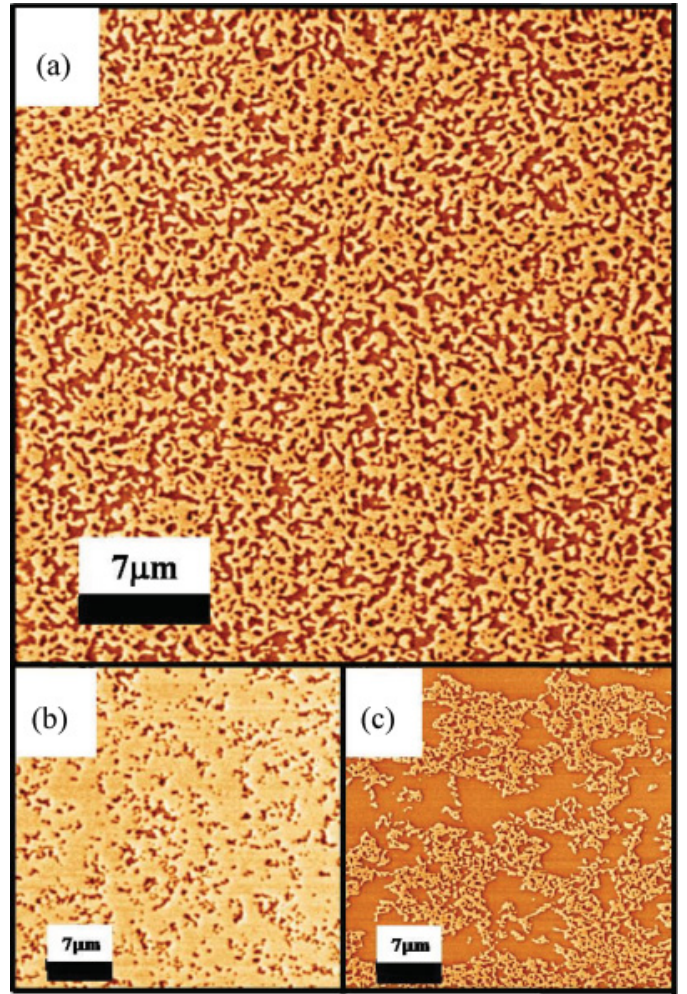

FIG. 2. (Color online) Domain structure of the HL at remanence. (a) Virgin domain state of the HL; (b) HL partially saturated in the direction of $m_{\mathrm{HL}}$ (virgin) (50\% of HL saturation moment); (c) HL partially saturated opposite to the direction of $m_{\mathrm{HL}}$ (virgin) (20\% of HL saturation moment).

In order to quantify the influence of the HL domain patterns on the SL loop-integrated asymmetry, the HL-induced dipolar stray field is calculated using HL saturation magnetization of $M_{\mathrm{s}}=170 \mathrm{kA} / \mathrm{m}$ and HL anisotropy constant of $K_{\mathrm{u}}=$ $550 \mathrm{~kJ} / \mathrm{m}^{3}$, both of which were extracted from in-plane and out-of-plane magnetometry measurements (Fig. 3).

The exchange constant was taken from data in literature ${ }^{22,23}$ to be $6.210^{-12} \mathrm{~J} / \mathrm{m}$. Using these values, a DW structure has

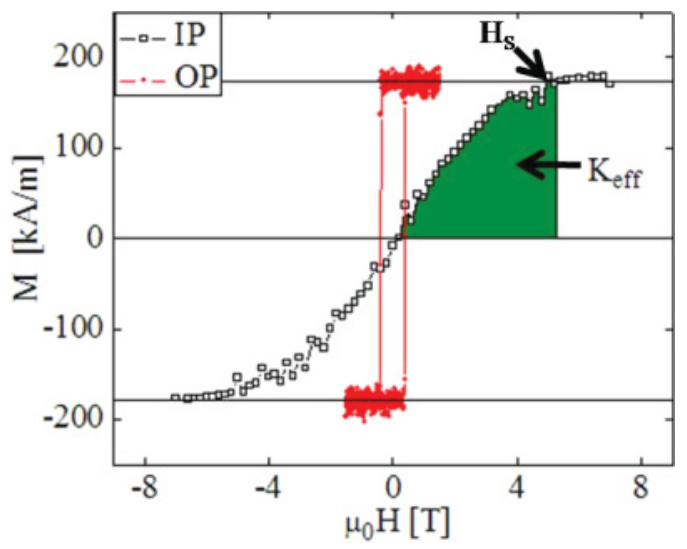

FIG. 3. (Color online) SQUID magnetometry in-plane (IP) and out-of-plane (OP) hysteresis loop for a $\mathrm{Co}_{74} \mathrm{~Tb}_{26}(20 \mathrm{~nm})$ film. The effective anisotropy constant $K_{\text {eff }}=K_{\mathrm{u}}-1 / 2 \mu_{0} M_{\mathrm{S}}{ }^{2}$ can be approximated as the surface under the IP loop between the origin and the saturation field $H_{\mathrm{S}}$. been computed using $\mathrm{OOMMF}^{24}$ in order to extract the DW width. From here we define $x$ and $y$ as in the film plane direction and $z$ perpendicular to it. In order to get an analytic expression of the DW stray field, a good approximation consists to assume that the variation of the $z$ component of the magnetization profile along the $x$ direction, i.e., perpendicular to the DW, has an arctangent form $\tan ^{-1}(x / \delta) .{ }^{25}$ Adjusting the arctangent profile to the profile calculated by OOMMF, a $\delta=$ $2 \mathrm{~nm}$ could be extracted. In the case of a single DW in a sample of thickness $h$, an effective DW width $\delta$, and a saturation magnetization $M_{\mathrm{S}}$, the dipolar stray field $H_{z}$ perpendicular to the sample surface at a distance $z$ over the surface is given by ${ }^{26}$

$$
H_{z}(x, z)=-4 M_{s} \tan ^{-1}\left(\frac{x h}{(z+\delta)(z+\delta+h)+x^{2}}\right) .
$$

As observed by MFM, the HL saturation corresponds to the shrinking of clusters with the original domain pattern. We model the stray field emitted by a cluster of domains as the sum of the stray fields emitted by the DWs using the previous equation. In order to take the $z$-dependence of the dipolar field into account we calculate the average dipolar field inside the 20-nm-thick free layer, which corresponds to a distance between 3.5 and $23.5 \mathrm{~nm}$.

The considered constant domain size is $0.5 \mu \mathrm{m}$, which corresponds approximately to the median diameter of the domains of the virgin state.

The resulting stray fields originating from 19 domains as a representative number for (a) many domains, (b) 5 domains, (c) 3 domains, and (d) single domain in a positive saturated red sample are shown in Fig. 4.

The amplitude and shape of stray field generated on top of one domain depends on the number of neighboring domains [Figs. 4(a)-4(d)]. Such a peculiar and subtle profile arises from the reduced size of the domains. When the domain structure is homogeneous, i.e., includes a large number of neighboring domains, the stray-field profile is symmetric. This can be seen in Fig. 4(a) where the stray field is simulated using a large number of domains (19). At the center of the domain's pattern, around position zero, the stray field $H_{z}$ has the same positive and negative amplitudes. Only the border of the domain pattern, especially the last DW, induces larger negative values of $H_{z}$ than positive ones. This asymmetry is enhanced when only few domains are considered, as shown in Figs. 4(b)-4(d). As a result, the negative stray field of a single negative domain is much larger than the positive stray field generated at the DW position [Fig. 4(d)]. In the light of those conclusions, the asymmetry of the minor SL cycle can be explained by imaging and comparing SL and HL domain patterns during ascending and descending branch of the SL hysteresis loop.

Let us first discuss the case of the "down" reversal. The SL has been saturated up and a negative field is applied [Fig. 5(a)]. The large reversed areas of the SL appear in brown, while nonreversed areas appear in light brown. The small domains appearing in dark brown are domains in the HL. As observed on Fig. 5(a), the reversal of the SL happens in regions of low density domains in the HL, and the propagation of the DWs is pinned by regions of high DW density in the HL. The previously mentioned modeling results show that regions with 
a low DW density exhibit the strongest negative dipolar field. Therefore, the reversal of the SL occurs as expected in regions where the effective negative applied field is the largest. After nucleation, the SL DWs propagate until reaching the border of a region with high HL domain density. This is observed in Figs. 5(a) and 5(b). A closer analysis of the MFM image shows that all the small domains at the periphery of the reversed domain in SL have the darker contrast, unlike the HL domains in the nonreversed SL region that have an intermediate contrast [inset of Figs. 5(b) and 5(c)]. This indicates that the reversed domains in the SL are blocked by domains in HL, as depicted in Fig. 5(d).

Let us now discuss the case of the up reversal. The SL is first saturated down, and a positive field is then applied such that up domains nucleate in the SL [Fig. 6(a)]. The reversed areas of the SL appear in light brown, while nonreversed areas appear in brown. Figure 6 shows that SL reversal starts in regions with no $\mathrm{HL}$ domains and that the propagation of the DW is blocked as soon as it reaches HL domains location.

The previously mentioned calculations show that the positive stray field is the highest in regions where multiple domains in the HL exist. Therefore one could have expected SL nucleation to occur in these regions.

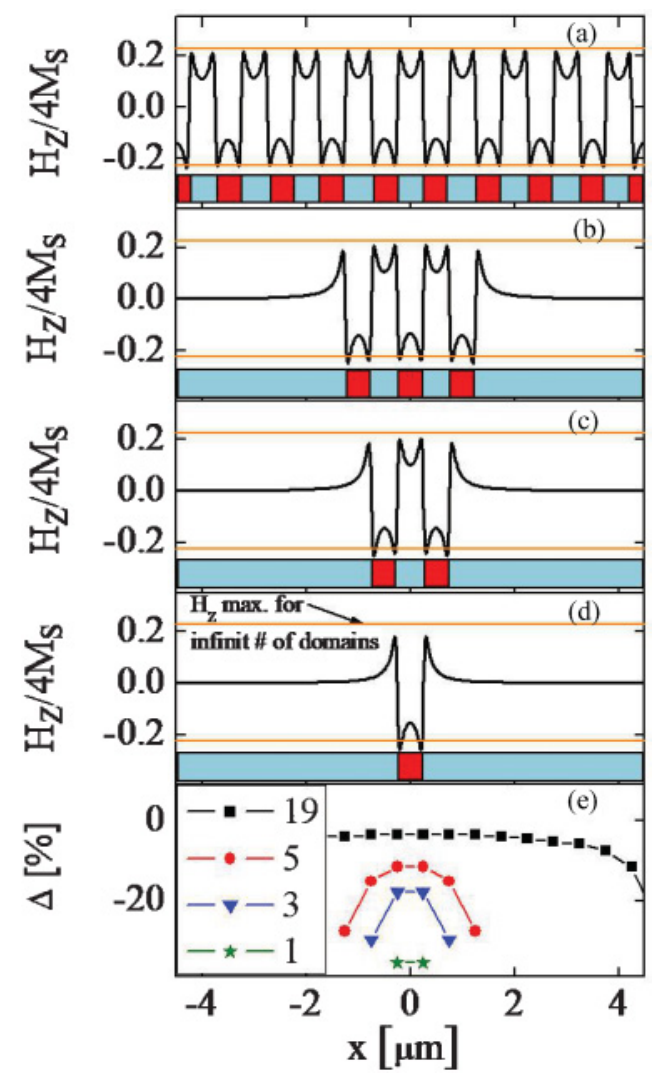

FIG. 4. (Color online) Calculated stray fields originating from a cluster of (a) 19 domains, (b) 5 domains, (c) 3 domains, and (d) single domain in a positive saturated blue sample. Red domains have negative magnetization; blue domains have positive magnetization. (e) Asymmetry $\Delta$ of the stray field close to a domain wall (DW) $\Delta=$ (maximum positive amplitude - maximum negative amplitude of the stray field) as a function of the distance from the center of the cluster.

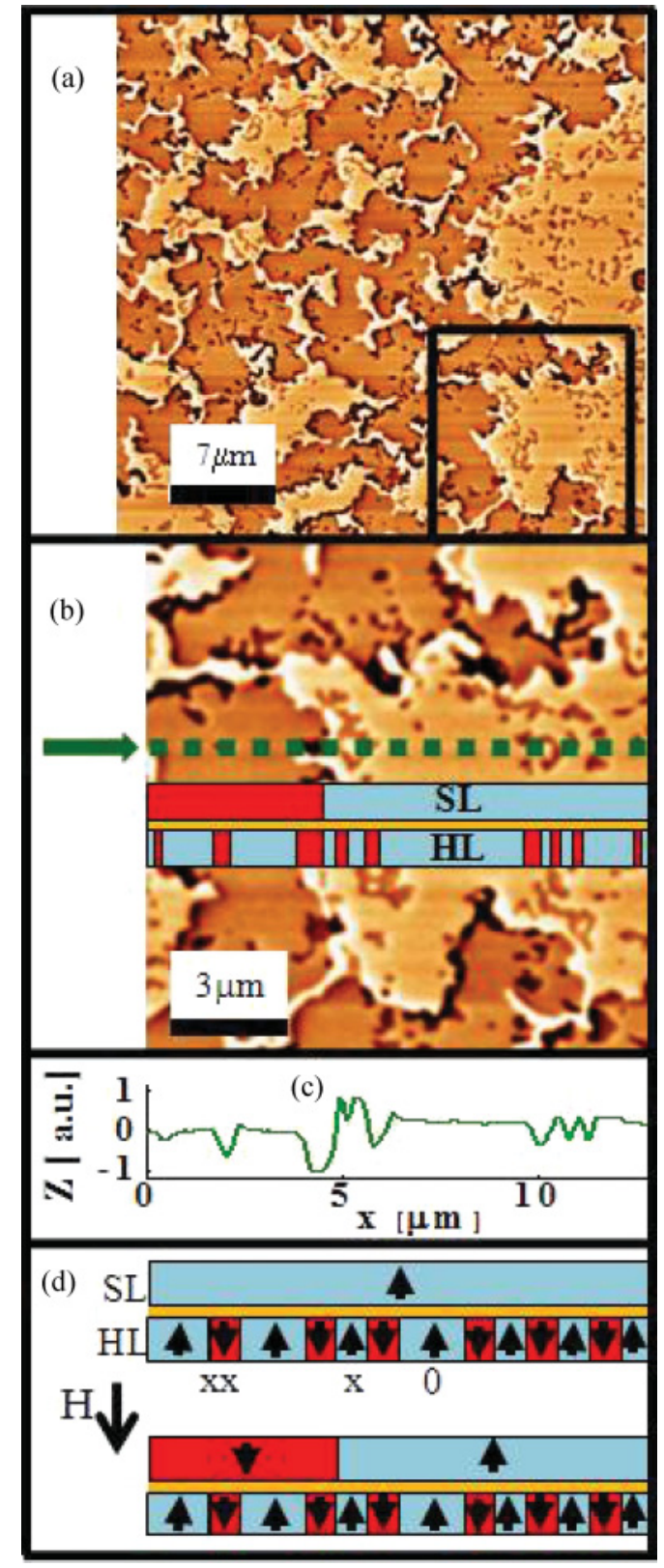

FIG. 5. (Color online) (a) MFM image of the remanent domain structure during down reversal. (b) Zoom into previous image. Inset: Sketch of the domain structure in soft layer (SL) and hard layer (HL) along an arbitrarily chosen green line of the MFM scan reconstructed from (c) the contrast $Z$ of the image along same line. (d) Sketch of the reversal process.

Nucleation of DWs should then preferentially occur in regions labeled XX in Fig. 6(d). However, no nucleation could be observed experimentally and even if nucleation occurred, no propagation takes place. Furthermore, considering the higher saturation magnetization of $M_{\mathrm{s}}=580 \mathrm{kA} / \mathrm{m}$ of the $\mathrm{Co}_{88} \mathrm{~Tb}_{12}$ $\mathrm{SL}$, the stability diameter of domains in the SL is higher than in the HL. This means that the locally confined HL stray field is insufficient to cause SL domain nucleation.

However, nucleation in regions labeled $\mathrm{X}$ will lead to propagation. As a consequence, SL reversal occurs first in a region without $\mathrm{HL}$ domains, and domain grows until the DW 


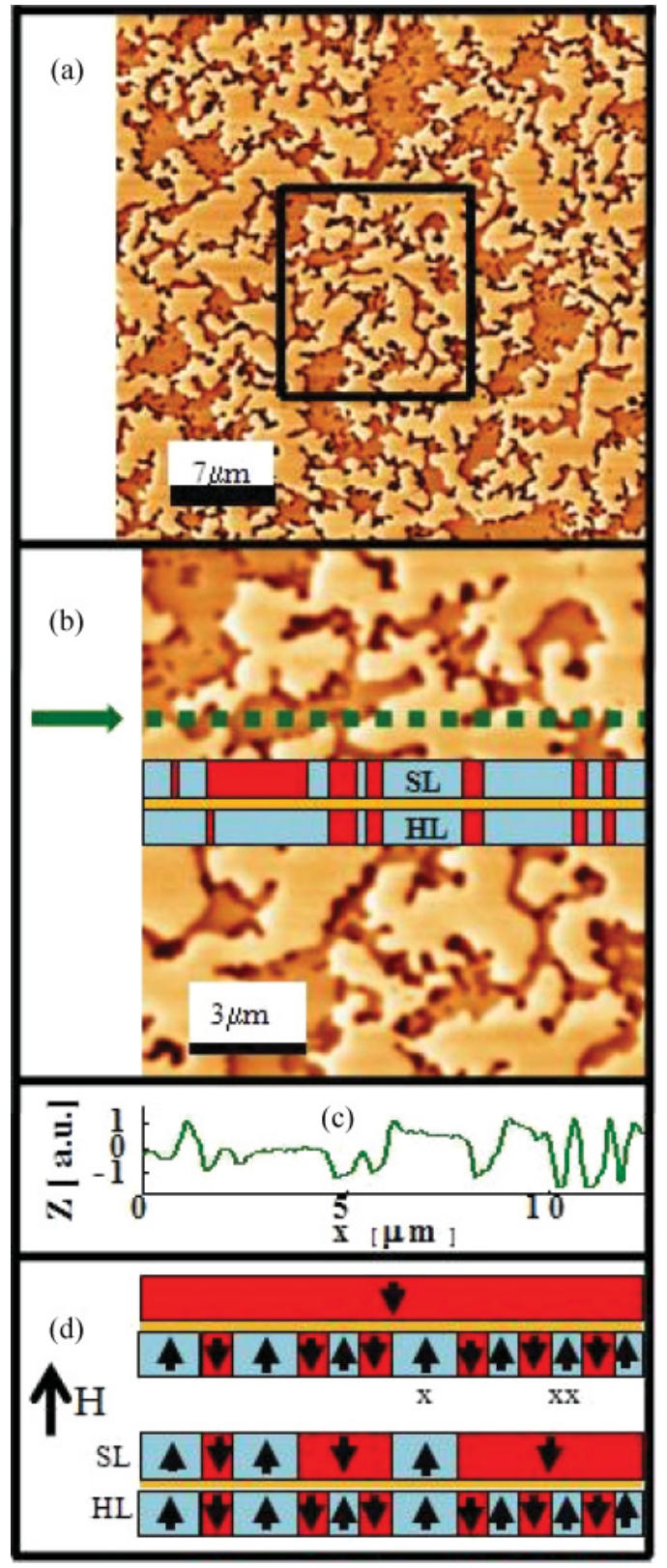

FIG. 6. (Color online) (a) MFM image of the remanent domain structure during up reversal. (b) Zoom into previous image. Inset: Sketch of the domain structure in soft layer (SL) and hard layer (HL) along an arbitrarily chosen line of the MFM scan reconstructed from (c) the contrast $Z$ of the image along same line. (d) Sketch of the reversal process.

meets a potential barrier, that are here given by the nearest reversed domains in the HL. Therefore, reversed domains in SL will preferentially cover regions without DWs in the HL.
This is in accordance to the observed MFM image [Figs. 6(a) and $6(b)]$.

\section{RESULT}

Based on the qualitative understanding of the SL domain nucleation and DW pinning processes obtained by MFM images and calculations, the asymmetry of the SL minor loop can now be explained.

In the case of down reversal of the SL, nucleation occurs in the SL in regions on top of HL region with low-density domains. SL DWs have then to propagate across a potential profile created by solely high density DW areas in the HL. In the case of up reversal of the SL, nucleation occurs in the SL in regions on top of an HL region without domains. SL DWs have then to propagate across a potential profile created by both high density DW areas and low density DW areas of the HL. The difference between up and down reversal arises then from the low density DW areas: in one case, they promote the nucleation of domains in the SL (down reversal); in the other case (up reversal), the low density DW areas are the location of the highest local fields that oppose to the propagation of the SL DWs and so cause a higher resistance towards saturation leading to a larger saturation field. Indeed, from our modeling, the stray field has been shown to be higher in those areas. Then, SL saturation needs a high applied field. The integrated asymmetry value shown in Fig. 1(d) is then explained by a variation of the density of single domain patterns in the HL as a function of field. With increasing HL saturation this density of single domain patterns increases, reaches a maximum, and decreases towards zero for the full saturation of the HL magnetization. Consequently the asymmetry $S$ in Fig. 1(d) increases first when increasing HL saturation reaches a maximum and disappears when the HL is saturated.

\section{CONCLUSION}

In conclusion the dipolar coupling between a SL and a HL in a $\mathrm{TbCo} / \mathrm{Cu} / \mathrm{TbCo}$ trilayer is shown to influence strongly the magnetization reversal inside the free layer. Despite the fact that the magnetization of TbCo alloys is low compared to "usual" transition metal compounds, the effect of the dipolar coupling is nevertheless strong enough to cause hysteresis loop asymmetry. From those results one could imagine using a generated stray field in order to control DW displacement, as described by Ref. 27, which might become relevant for applications such as the race track memory. ${ }^{28}$

\section{ACKNOWLEDGMENTS}

A.B. acknowledges funding from the Basque Government under Program No. PI2009-17 and the Spanish Ministry of Science and Education under Project No. MAT2009-07980. This work is supported by the ANR-NSF FRIENDS Project.
*Present address: Center for Magnetic Recording Research, 9500 Gilman Dr., La Jolla, CA 92093-0401, USA; mgottwald@ucsd.edu ${ }^{1}$ W. H. Meiklejohn and C. P. Bean, Phys. Rev. 102, 1413 (1956).
${ }^{2}$ J. Nogués and I. K. Schuller, J. Magn. Magn. Mater. 192, 203 (1999).

${ }^{3}$ P. Blomqvist, K. M. Krishnan, and H. Ohldag, Phys. Rev. Lett. 94, 107203 (2005). 
${ }^{4}$ J. Camarero, J. Sort, A. Hoffmann, J. M. García-Martín, B. Dieny, R. Miranda, and J. Nogués, Phys. Rev. Lett. 95, 057204 (2005).

${ }^{5}$ A. Berger, S. Mangin, J. McCord, O. Hellwig, and E. E. Fullerton, Phys. Rev. B 82, 104423 (2010).

${ }^{6}$ O. Fruchart, N. Rougemaille, A. Bendounan, J.-C. Toussaint, R. Belkhou, Y. Tian, H. Yu, F. Cheynis, A. Masseboeuf, P. Bayle-Guillemaud, and A. Marty, IEEE Trans. Magn. 46, 1552 (2010).

${ }^{7}$ J. Briones, F. Montaigne, D. Lacour, M. Hehn, M. Carrey, and J. Childress, Appl. Phys. Lett. 92, 32508 (2008).

${ }^{8}$ N. Rougemaille, F. Montaigne, B. Canals, A. Duluard, D. Lacour, M. Hehn, R. Belkhou, O. Fruchart, S. El Moussaoui, A. Bendounan, and F. Maccherozzi, Phys. Rev. Lett. 106, 057209 (2011).

${ }^{9}$ S. Mangin, T. Hauet, P. Fischer, D. H. Kim, J. B. Kortright, K. Chesnel, E. Arenholz, and E. E. Fullerton, Phys. Rev. B 78, 024424 (2008).

${ }^{10}$ P. J. Metaxas, P.-J. Zermatten, J.-P. Jamet, J. Ferre, G. Gaudin, B. Rodmacq, A. Schuhl, and R. L. Stamps, Appl. Phys. Lett. 94, 132504 (2009).

${ }^{11}$ A. Berger, D. T. Margulies, and H. Do, Appl. Phys. Lett. 85, 1571 (2004).

${ }^{12}$ A. Berger, O. Hovorka, G. Friedman, and E. E. Fullerton, Phys. Rev. B 78, 224407 (2008).

${ }^{13}$ O. Hellwig, A. Berger, T. Thomson, E. Dobisz, Z. Z. Bandic, H. Yang, D. S. Kercher, and E. E. Fullerton, Appl. Phys. Lett. 90, 162516 (2007).

${ }^{14}$ M. Albrecht, G. Hu, A. Moser, O. Hellwig, and B. D. Terris, J. Appl. Phys. 97, 103910 (2005).
${ }^{15}$ S. Gider, B-U. Runge, A. C. Marley, and S. S. P. Parkin, Science 281, 797 (1998).

${ }^{16}$ I. Tudosa, J. A. Katine, S. Mangin, and E. E. Fullerton, Appl. Phys. Lett. 96, 212504 (2010).

${ }^{17} \mathrm{~S}$. Wiebel, J. P Jamet, N. Vernier, A. Mougin, J. Ferré, V. Baltz, B. Rodmacq, and B. Dieny, Appl. Phys. Lett. 86, 142502 (2005).

${ }^{18}$ T. Hauet, C. M. Gunther, B. Pfau, M. E. Schabes, J.-U. Thiele, R. L. Rick, P. Fischer, S. Eisebitt, and O. Hellwig, Phys. Rev. B 77, 184421 (2008)

${ }^{19}$ B. Rodmacq, V. Baltz, and B. Dieny, Phys. Rev. B 73, 092405 (2006).

${ }^{20}$ S. Mangin, D. Ravelosona, J. A. Katine, M. J. Carey, B. D. Terris, and E. E. Fullerton, Nature Mat. 5, 210 (2006).

${ }^{21}$ I. Yulaev, M. Lubarda, S. Mangin, V. Lomakin, and E. E. Fullerton, Appl. Phys. Lett. 99, 132502 (2011).

${ }^{22}$ Z. Q. Zou, H. Wang, and C. Yu, J. Appl. Phys. 93, 5268 (2003).

${ }^{23}$ R. Hasegawa, J. Appl. Phys. 45, 3109 (1974).

${ }^{24}$ M. J. Donahue and D. G. Porter, Interagency Report No. NISTIR 6376, National Institute of Standards and Technology, Gaithersburg, MD 1999, http://math.nist.gov/oommf.

${ }^{25}$ D. Rugar, H. J. Mamin, P. Guethner, S. E. Lambert, J. E. Stern, I. McFadyen, and T. Yogi, J. Appl. Phys. 68, 1169 (1990).

${ }^{26}$ M. Hehn, S. Padovani, K. Ounadjela, and J. P. Bucher, Phys. Rev. B 54, 3428 (1996).

${ }^{27}$ S. Park, N. M. Nguyen, C. Burrowes, E. E. Fullerton, C. Chappert, L. Prejebeanu, F. Garcia-Sanchez, and D. Ravelosona, Appl. Phys. Lett. 98, 232512 (2011).

${ }^{28}$ S. S. P. Parkin, M. Hayashi, and L. Thomas, Science 320, 190 (2008). 